Recepción: 20 / 04 / 2017

Aceptación: 20 / 05 / 2017

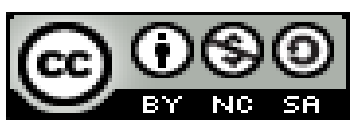

Ciencias Químicas

Publicación: 15 / 07 / 2017

Artículo Científico

\title{
Evaluación de resultados fisicoquímicos y microbiológicos del agua potable expendida en tanqueros
}

\section{Evaluation of physicochemical and microbiological results of drinking water issued in tankers}

\section{Os resultados da avaliação de água emitido petroleiros físico-químicos e microbiológicos}

\author{
Madeleen P. Cruz-Vargas I \\ made_pao91@hotmail.com \\ Walter E. Mariscal-Santi II \\ walter.mariscals@ug.edu.ec \\ Nancy A. Sorroza-Rojas III \\ nancysorroza@uees.edu.ec
}

\author{
Nancy E. Villegas-Villao ${ }^{\mathrm{IV}}$ \\ nanvillegas@hotmail.com \\ Frella S. García-Larreta v \\ nanvillegas@hotmail.com \\ Raisa S. Mariscal-García VI \\ raisamariscal@hotmail.com
}

Correspondencia: made_pao91@hotmail.com

I. Docente, Universidad de Guayaquil, Guayaquil, Ecuador.

II. Magister en Diseño Curricular; Diplomado en Docencia Superior; Químico y Farmacéutico; Químico y Farmacéutico; Universidad de Guayaquil, Guayaquil, Ecuador.

III. Magister en Bioquímica Clínica; Química y Farmacéutica; Universidad de Especialidades Espíritu Santo, Samborondón, Ecuador.

IV. Especialista en Biotecnología, Biología Molecular e Ingeniería Genética; Magister en Diseño Curricular; Doctora en Medicina y Cirugía; Universidad de Guayaquil, Guayaquil, Ecuador.

v. Magister n Diseño Curricular; Diplomado en Docencia Superior; Química y Farmacéutica; Universidad de Guayaquil, Guayaquil, Ecuador.

VI. Medica General, Universidad de Guayaquil, Guayaquil, Ecuador. 
Madeleen P. Cruz-Vargas; Walter E. Mariscal-Santi; Nancy A. Sorroza-Rojas; Nancy E. Villegas-Villao; Frella S. GarcíaLarreta; Raisa S. Mariscal-García

\section{Resumen}

El agua es un elemento vital para los seres humanos, por esta razón es es importante mantener la calidad de esta en óptimas condiciones para garantizar la ausencia de componentes contaminantes durante su distribución. El presente trabajo de titulación tuvo como objetivo Evaluar la Calidad Fisicoquímica y Microbiológica del agua potable distribuida en tanqueros en la Parroquia El Morro del cantón Guayaquil. Los análisis se realizaron durante los meses de febrero y marzo del 2017, durante este periodo se analizaron nueve muestras en diferentes zonas de la parroquia El Morro. Se determinó la presencia de Coliformes fecales en todos los sectores siendo el sector Norte Los Pocitos el más representativo donde la muestra T2 presentó un valor de $1.22 \mathrm{UFC} / 100 \mathrm{ml}$ y se evidenció que el Cloro Residual no cumplía en ningún sector con el mínimo establecido en la NORMA INEN 1108:2014, motivo por el cual el agua expendida por los tanqueros en la Parroquia El Morro no se considera apta para el consumo humano. Por ser un patrimonio nacional estratégico de uso público EL AGUA POTABLE deberá cumplir con los criterios de calidad en base a parámetros físicos, químicos y microbiológicos tal como lo establece la NORMA INEN 1108:2014.

Palabras claves: Tanquero; calidad; agua; coliformes fecales. 


\section{Summary}

Water is a vital element for humans, for this reason it is important to maintain the quality of the water in optimal conditions to guarantee the absence of contaminating components during its distribution. The objective of this study was to evaluate the physical and chemical quality of drinking water distributed in tankers in the El Morro Parish of Guayaquil. The analyzes were carried out during the months of February and March of 2017, during this period were analyzed nine samples in different areas of the parish El Morro. The presence of fecal coliforms was determined in all sectors, with the northern sector being the most representative where the sample T2 presented a value of $1.22 \mathrm{CFU} / 100 \mathrm{ml}$ and it was evidenced that the Residual Chlorine did not comply in any sector with the minimum established in the NORMA INEN 1108: 2014, reason why the water expended by the tankers in the Parroquia El Morro is not considered suitable for human consumption. Because it is a strategic national patrimony of public use, POTABLE WATER must comply with the quality criteria based on physical, chemical and microbiological parameters as established in NORMA INEN 1108: 2014.

Key words: Tanker; quality; water; fecal coliforms. 
Madeleen P. Cruz-Vargas; Walter E. Mariscal-Santi; Nancy A. Sorroza-Rojas; Nancy E. Villegas-Villao; Frella S. GarcíaLarreta; Raisa S. Mariscal-García

\section{Resumo}

A água é um elemento essencial para o ser humano, por esta razão, é importante para manter a qualidade desta, em condições óptimas para garantir a ausência de contaminação dos componentes durante a distribuição. Este trabalho foi avaliar Físico grau e qualidade microbiológica da água potável distribuída por meio de cisternas na Freguesia El Morro Canton Guayaquil. As análises foram realizadas durante os meses de fevereiro e 2017 de março, durante este período de nove amostras foram analisadas em diferentes áreas da paróquia El Morro. a presença de coliformes fecais foi determinada em todos os sectores, sendo o sector norte Os Pocitos a mais representativa, onde a amostra T2 apresentaram um valor de 1,22 UFC / $100 \mathrm{~mL}$ e mostrou que o cloro residual foi, em qualquer sector com um mínimo estabelecido no NORMA inen 1108: 2014, por despendido por tanques de água no Parroquia El Morro não é considerado adequado para consumo humano. Como um ativo nacionais estratégicos para uso público ÁGUA POTÁVEL devem atender a critérios de qualidade com base em parâmetros físicos, químicos e microbiológicos previstos por inen Regra 1108: 2014.

Palavras-chave: Tanquero; qualidade; água: coliformes fecais. 


\section{Introducción.}

El agua potable es esencial para el bienestar y seguridad de la población mundial ya que una gran parte de esta carece de este líquido vital, en el Ecuador aún hay localidades rurales en las cuales la dispensación del agua potable es deficiente esta se determina comparando las características fisicoquímicas y microbiológicas de una muestra bajo estándares de calidad de manera puntual para el agua potable y garantizar el suministro de agua limpia y saludable para el consumo humano. (Becerra Ramírez \& Salas Benítez, 2016) (Arrojo, 2006)

En el plan de desarrollo del GAD realizado en el 2015 en la Parroquia El Morro se encontró 314 casos de hogares que no reciben agua potable por tuberías sino por Tanqueros (que equivalen al 26.75\% de esa población). (Chiles Arévalo, 2015)

El agua potable proviene del rio Guayas, pasa por los canales de Don Juan y luego son tratadas en la planta de San Antonio donde pasan por procesos de potabilización y por tuberías llega hasta la toma de agua de Engabao donde los tanqueros se abastecen para distribuir a los diferentes sectores entre esos la parroquia de El Morro. (Aricoché, 2012)

El trabajo a realizar es una evaluación de los resultados físico-químicos y microbiológicos establecidos por la NORMA INEN1108:2014 de requisito para determinar si la calidad del agua potable que se expende en tanqueros en la Parroquia El Morro será para determinar si es apta para el consumo humano. (Ramos Castellanos, 2007)

La organización mundial de la salud y el fondo de las naciones unidas para la infancia la definen como el agua utilizada para los fines domésticos y la higiene personal, así como para beber y cocinar siendo el agua potable salubre aquella en que sus características microbianas, químicas y 
Madeleen P. Cruz-Vargas; Walter E. Mariscal-Santi; Nancy A. Sorroza-Rojas; Nancy E. Villegas-Villao; Frella S. GarcíaLarreta; Raisa S. Mariscal-García

físicas cumplen con las pautas de la OMS o los patrones nacionales sobre la calidad del agua potable. (Rodriguez, 2008)

Se denomina agua potable, al agua que puede ser consumida sin restricción, no causa daño a la salud del usuario y sus características físicas, químicas y microbiológicas han sido tratadas a fin de garantizar su aptitud para el consumo humano.

Por tanto el agua no potable es aquella que podría causar daño a la salud del usuario y no cumple con los requisitos físico-químicos y microbiológicos establecidos por la norma nacional.

\section{Agua en El Morro}

La Parroquia El Morro está ubicada al sureste de Guayaquil; a unos 90 km de esta ciudad, y cercana a las poblaciones General Villamil Playas y Posorja; El Morro limita al norte con la parroquia Juan Gómez Rendón, al sur con la parroquia Posorja, al este con la parroquia Juan Gómez Rendón y el Golfo de Guayaquil y al oeste con el cantón General Villamil Playas. (GAD, 2015)

El Golfo de Guayaquil forma un área geográfica y biótica de reconocida importancia para el país. Por el lado del canal del Morro penetra el agua salada al territorio continental a través de una serie de esteros, cuyos meandros constituyen un importante sistema estuarino. (GAD, 2015)

Los esteros y el área de mar adyacente al Golfo de Guayaquil, son áreas altamente productivas debido a la presencia de los eventos fríos de "La Niña" y los cálidos eventos de "El Niño- Oscilación Sur" que permiten el desarrollo de organismos acuáticos, de interés para la pesca artesanal costera y a su vez para la conservación de la biodiversidad. 
Respecto a la procedencia principal del agua, un 73,25\% de la población la recibe mediante red pública, mientras que el otro porcentaje restante mayormente ubicados en recintos y caseríos con un $14,31 \%$ obtiene esta fuente vital a través de carros repartidores, y en menor porcentaje la obtiene de pozos, y de otras fuentes varias.

Tabla $\mathbf{N}^{\circ}$ 1. Procedencia principal del agua recibida en El Morro

\begin{tabular}{|l|l|l|}
\hline Procedencia principal del agua recibida & Casos & $\%$ \\
\hline De red pública & 860 & $73,25 \%$ \\
\hline De pozo & 86 & $7,33 \%$ \\
\hline De río, vertiente, acequia o canal & 3 & $0,26 \%$ \\
\hline De carro repartidor (tanqueros) & 168 & $14,31 \%$ \\
\hline Otro (Agua lluvia/albarrada) & 57 & $4,86 \%$ \\
\hline Total & 1174 & $100,00 \%$ \\
\hline
\end{tabular}

Fuente: INEC 2010

\section{Norma Técnica Ecuatoriana NTE INEN 1108:2014}

Esta norma establece los requisitos que debe cumplir el agua potable para consumo humano, los sistemas de abastecimiento de agua potable deberían acogerse al Reglamento de buenas prácticas de Manufactura (producción) del Ministerio de Salud Pública. (INEN, 2014)

El manejo y conservación de las muestras para la realización de los análisis debe realizarse de acuerdo con lo establecido en los métodos estandarizados para el agua potable y residual (Standard Methods). 
Madeleen P. Cruz-Vargas; Walter E. Mariscal-Santi; Nancy A. Sorroza-Rojas; Nancy E. Villegas-Villao; Frella S. GarcíaLarreta; Raisa S. Mariscal-García

\section{Algunas Características Generales Del Agua}

Se considera que las propiedades físicas y químicas del agua son las responsables de que la Tierra sea tal como se conoce y que la vida misma es consecuencia de las propiedades tan especiales de la molécula de agua, ya que se considera que las primeras formas primitivas de vida comenzaron en una solución acuosa.

La mayor parte es salubre y una parte muy pequeña es agua dulce.

Contribuye a mantener el clima en la Tierra, disuelve a una gran cantidad de sustancias, que pueden llegar a ser contaminantes, y es esencial para las formas de vida conocidas en la Tierra.

El agua se presenta principalmente como un líquido de características poco comunes, es un recurso natural indispensable para todos los seres vivos y en general forma parte de toda la materia viva.

El ser humano la utiliza para realizar muchas de sus actividades como la agricultura, la industria, el transporte y otras actividades diarias.

\section{Parámetros Físicos: color, turbiedad, sabor}

\section{Sólidos Totales Disueltos}

El agua puede contener tanto partículas en suspensión como compuestos solubilizados, definiéndose la suma de ambos como sólidos totales, lo constituyen las sales que se encuentran presentes y que no pueden ser separados del líquido por algún medio físico.

Está relacionada con la conductividad eléctrica, se mide en ppm y se determinan gravimétricamente mediante filtración, vacío o presión 


\section{Parámetros Químicos}

Los parámetros químicos están relacionados con la capacidad del agua para disolver diversas sustancias entre las que podemos mencionar a los sólidos disueltos totales, alcalinidad, dureza, fluoruros, materias orgánicas y nutrientes.

\section{Estimación del Índice de Calidad de Agua General "ICA"}

El "ICA" adopta para condiciones óptimas un valor máximo determinado de 100, que va disminuyendo con el aumento de la contaminación el curso de agua en estudio.

\section{Criterios de calidad para aguas de consumo humano y uso doméstico}

Se entiende por agua para consumo humano y uso doméstico aquella que es obtenida de cuerpos de aguas superficiales o subterráneas, y que luego de ser tratada será empleada por individuos o comunidades en actividades como:

a) Bebida y preparación de alimentos para consumo humano,

b) Satisfacción de necesidades domésticas, individuales o colectivas, tales como higiene personal y limpieza de elementos, materiales o utensilios,

Esta Norma aplica a la selección de aguas captadas para consumo humano y uso doméstico, que únicamente requieran de tratamiento convencional, para lo cual se deberán cumplir con los criterios indicados en la siguiente 


\section{Metodología.}

El trabajo se desarrolló mediante un diseño de investigación Analítico - Descriptivo de corte transversal mediante la Norma Ecuatoriana INEN 1108:2014 de requisito y el aporte de información encontrada en el GAD 2015 (Gobierno Autónomo Descentralizados) de la Parroquia El Morroen en los meses de febrero a marzo del 2017, se consideró a los tanqueros que distribuyen el agua a los diferentes sectores de El Morro.

Tabla $\mathbf{N}^{\circ}$ 2. Examenes fisicoquimicos

\begin{tabular}{|c|c|c|c|}
\hline Parámetro & Equipo & Marca & Metodología \\
\hline Arsénico & \begin{tabular}{|ll}
\multicolumn{2}{|c|}{ Espectrofotómetro } \\
de & Absorción \\
Atómica & \\
\end{tabular} & Thermo Fisher Scientific & St. Methods Ed. 22 \\
\hline Cadmio & \begin{tabular}{|ll}
\multicolumn{2}{|c}{ Espectrofotómetro } \\
de & Absorción \\
Atómica &
\end{tabular} & Thermo Fisher Scientific & St. Methods Ed. 22 \\
\hline Cobre & \begin{tabular}{|ll}
\multicolumn{2}{|c|}{ Espectrofotómetro } \\
de & Absorción \\
Atómica & \\
\end{tabular} & Thermo Fisher Scientific & St. Methods Ed. 22 \\
\hline Cromo & \begin{tabular}{|ll}
\multicolumn{2}{|c|}{ Espectrofotómetro } \\
de & Absorción \\
Atómica & \\
\end{tabular} & Thermo Fisher Scientific & St. Methods Ed. 22 \\
\hline Mercurio & \begin{tabular}{|ll}
\multicolumn{2}{|c}{ Espectrofotómetro } \\
de & Absorción \\
Atómica & \\
\end{tabular} & Thermo Fisher Scientific & St. Methods Ed. 22 \\
\hline Níquel & \begin{tabular}{|ll}
\multicolumn{2}{|c}{ Espectrofotómetro } \\
de & Absorción \\
Atómica & \\
\end{tabular} & Thermo Fisher Scientific & St. Methods Ed. 22 \\
\hline Plomo & \begin{tabular}{|l}
\multicolumn{2}{|c}{ Espectrofotómetro } \\
de $\quad$ Absorción \\
Atómica
\end{tabular} & Thermo Fisher Scientific & St. Methods Ed. 22 \\
\hline $\begin{array}{l}\text { Cloro Libre } \\
\text { Residual }\end{array}$ & Comparador de cloro & Orbeco - Hellige Technical & St. Methods Ed. 22 \\
\hline Fluoruro & Nova 60 & Merck & St. Methods Ed. 22 \\
\hline Nitratos & Nova 60 & Merck & St. Methods Ed. 22 \\
\hline Nitritos & Espectrofotómetro & Hach & St. Methods Ed. 22 \\
\hline Turbidez & Turbidimetro & Orbeco - Hellige Technical & St. Methods Ed. 22 \\
\hline
\end{tabular}


Tabla $\mathbf{N}^{\circ}$ 3. Examenes Microbiologicos

\section{Parámetro \\ Equipo \\ Coliformes fecales \\ Filtración por membrana}

Se recolectaron 9 muestras de tanqueros que abastecen a la Población, la muestra y tipo de muestra es probabilístico no aleatorio estratificado.

- Zona Norte: es la zona superior de estudio donde se obtendrán tres muestras de tanqueros diferentes que distribuyen en esta zona: Tanquero 1 (T1), Tanquero 2 (T2), Tanquero 3 (T3)

- Zona Sur: es la zona inferior en donde se obtendrán tres muestras de la misma forma que el sector norte. Tanquero 4 (T), Tanquero 5 (T5), Tanquero 6 (T6)

- Zona Centro: es la zona central del estudio donde se obtendrán tres muestras similares a los sectores anteriores. Tanquero 7 (T7), Tanquero 8 (T8), Tanquero 9 (T9)

Tabla $\mathbf{N}^{\circ}$ 4. Registro de recolección de muestras

\begin{tabular}{|c|c|c|c|}
\hline \multicolumn{4}{|c|}{ NÚMERO DE MUESTRAS } \\
\hline Id & Fecha & Hora de toma & Lugar de toma \\
\hline $\mathrm{T} 1$ & 27/02/2017 & $09: 15$ & Barrio Los Pocitos \\
\hline $\mathrm{T} 2$ & 27/02/2017 & $11: 45$ & Barrio Los Pocitos \\
\hline $\mathrm{T} 3$ & $27 / 02 / 2017$ & $14: 03$ & Barrio Los Pocitos \\
\hline $\mathrm{T} 4$ & $10 / 03 / 2017$ & 09:00 & Barrio Las Peñas \\
\hline T5 & $10 / 03 / 2017$ & $11: 26$ & Barrio Las Peñas \\
\hline T6 & $10 / 03 / 2017$ & $14: 17$ & Barrio Las Peñas \\
\hline $\mathrm{T} 7$ & 24/03/2017 & 09:10 & Barrio Tamarindo \\
\hline $\mathrm{T} 8$ & 24/03/2017 & $11: 36$ & Barrio Tamarindo \\
\hline T9 & 24/03/2017 & $14: 29$ & Barrio Tamarindo \\
\hline
\end{tabular}

Sector
Norte de la Parroquia
Norte de la Parroquia
Norte de la Parroquia
Sur de la Parroquia
Sur de la Parroquia
Sur de la Parroquia
Centro de la Parroquia
Centro de la Parroquia
Centro de la Parroquia

Observaciòn

Tanquero $\mathrm{N}^{\circ} 14$ Tanquero $\mathrm{N}^{\circ} 7$ Tanquero $\mathrm{N}^{\circ} 2$ Tanquero $\mathrm{N}^{\circ} 9$ Tanquero $\mathrm{N}^{\circ} 17$ Tanquero $\mathrm{N}^{\circ} 22$ Tanquero $\mathrm{N}^{\circ} 3$

Tanquero $\mathrm{N}^{\circ} 19$ Tanquero $\mathrm{N}^{\circ} 6$

Fuente: Madeleen Paola Cruz Vargas 2017 
Madeleen P. Cruz-Vargas; Walter E. Mariscal-Santi; Nancy A. Sorroza-Rojas; Nancy E. Villegas-Villao; Frella S. GarcíaLarreta; Raisa S. Mariscal-García

\section{Resultados.}

Una vez realizados los análisis respectivos a las muestras indicadas en el monitoreo se obtuvieron los siguientes resultados.

Tabla $\mathbf{N}^{\circ}$ 5. Indicador Microbiológico de la Calidad de Agua de tanqueros registrado en el Sector Norte de la Parroquia San Jacinto de El Morro Barrió Los Pocitos tomados en el Mes de Febrero del 2017: Parámetro "Coliformes Fecales"

$\begin{array}{cccc}\text { ID DE LA MUESTRA } & \text { RESULTADO } & \text { PARÀMETRO } & \text { UNIDAD } \\ \text { T1 } & 1.22 & <1 & \text { UFC/100 ML } \\ \text { T2 } & 1.12 & <1 & \text { UFC/100 ML } \\ \text { T3 } & 1.08 & <1 & \text { UFC/100 ML } \\ \text { MEDIA } & 1.14 & <1 & \text { UFC/100 ML } \\ \text { DESVIACIÒN } & & & \\ \text { ESTANDAR } & 0.07 & & \end{array}$

Fuente: Madeleen Cruz Vargas 2017

Los resultados obtenidos en la zona norte de la Parroquia El Morro sector Los Pocitos para Coliformes fecales se muestran positivos con un valor > $1 \mathrm{UFC} / 100 \mathrm{ml}$, por encima de los límites permisibles en base a la Norma INEN 1108:2014. Estableciendo que la muestra T1 tiene el valor más elevado con $1.22 \mathrm{UFC} / \mathrm{ml}$ y la muestra T3 tiene el valor más bajo con $1.08 \mathrm{UFC} / \mathrm{ml}$, con una media de $1.14 \mathrm{UFC} / \mathrm{ml}$ y una desviación estándar de 0.07 lo cual indica que los valores no están muy dispersos. El consumo de esta agua implica una probabilidad de adquirir enfermedades gastrointestinales causadas por bacterias.

El agua para el consumo humano proveniente de los tres tanqueros en base a la NORMA INEN 1108:2014 en su análisis microbiológico indica que los resultados obtenidos en el sector norte de la Parroquia El Morro presentan la ausencia del microorganismo Cryptosporidium 
Tabla $\mathbf{N}^{\circ}$ 6. Indicadores Físicos de la Calidad de Agua registrado en el Sector Norte de la Parroquia San Jacinto de El Morro Barrio Los Pocitos tomados en el Mes de Febrero del 2017

\begin{tabular}{|c|c|c|c|c|}
\hline \multirow{2}{*}{ Norte } & & \multicolumn{3}{|l|}{ Parámetro } \\
\hline & & Turbidez & Color & Olor \\
\hline & Unidad & Ntu & Utc & - \\
\hline & $\mathbf{T 1}$ & 0.80 & 0.00 & No objetable \\
\hline$\pi$ & $\mathbf{T} 2$ & 0.65 & 0.00 & No objetable \\
\hline 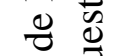 & T3 & 0.49 & 0.00 & No objetable \\
\hline ટ્己 & Media & 0.65 & 0 & \\
\hline & Desviación estándar & 0.16 & 0 & \\
\hline & Requisito & Máx 5 & Máx 15 & No objetable \\
\hline
\end{tabular}

Fuente: Madeleen Cruz Vargas 2017.

La muestra T1 tiene el valor más alto correspondiente a $0.80 \mathrm{Ntu}$ y la muestra T3 tiene el valor más bajo $0.49 \mathrm{Ntu}$ con una media de $0.65 \mathrm{NTu}$, cumpliendo con los requisitos determinados en la norma; de modo tal que la desviación estándar es 0.16 en la zona Norte. En cuanto al color y olor los resultados obtenidos están dentro de los requisitos permitidos por la NORMA INEN 1108:2014. 
Madeleen P. Cruz-Vargas; Walter E. Mariscal-Santi; Nancy A. Sorroza-Rojas; Nancy E. Villegas-Villao; Frella S. GarcíaLarreta; Raisa S. Mariscal-García

Tabla $\mathbf{N}^{\circ}$ 7. Indicadores Químicos de la Calidad de Agua de Tanqueros registrado en el Sector Norte de la Parroquia San Jacinto de El Morro Barrio Los Pocitos tomados en el Mes de Febrero del 2017

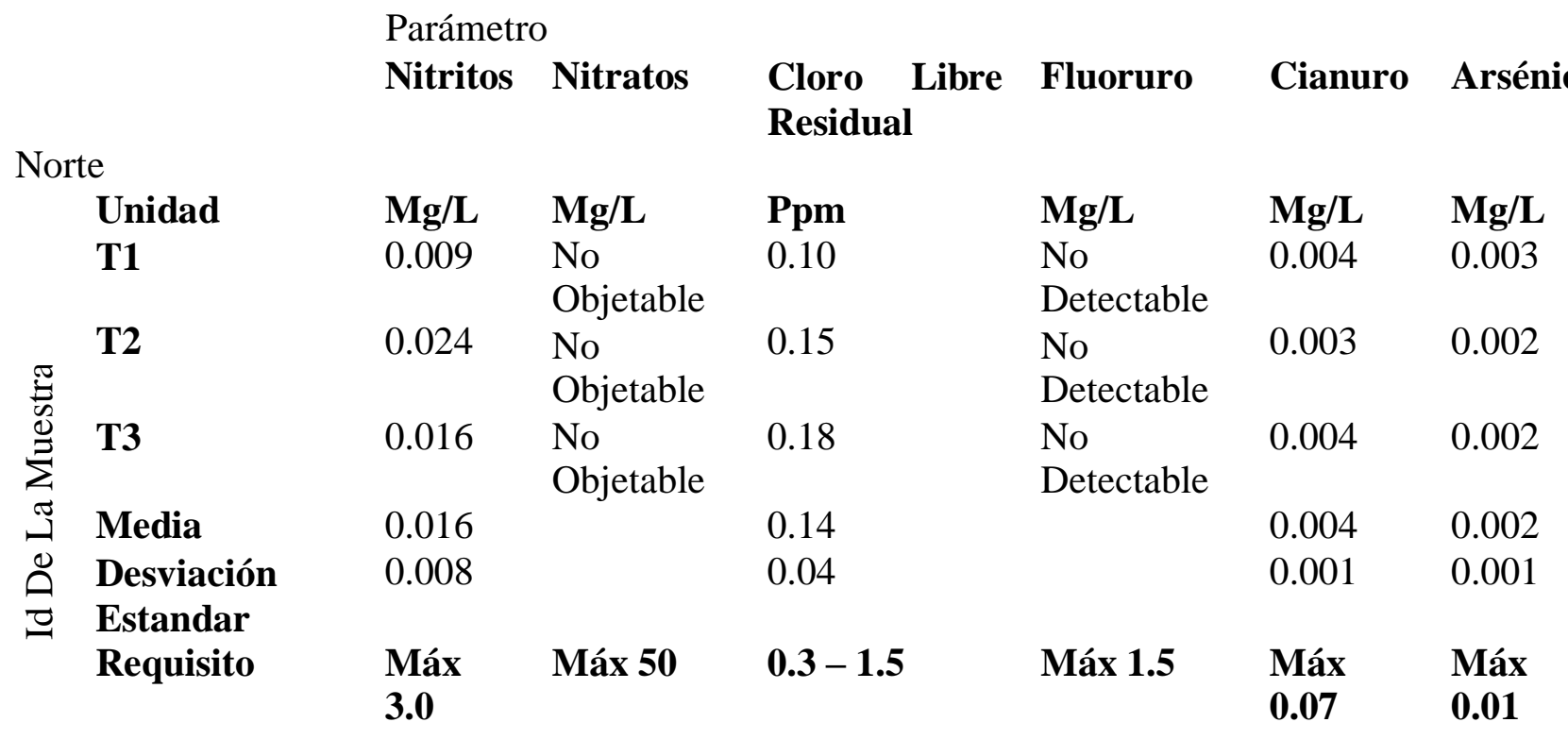

Fuente: Madeleen Cruz Vargas 2017

Los parámetros nitratos -no objetable- y fluoruros -no detectable- cumplen con la NORMA INEN 1108:2014. Para Nitritos todas las muestras analizadas cumplen con la norma; la muestra T2 muestra el valor más alto $0.024 \mathrm{mg} / \mathrm{L}$ mientras que la muestra $\mathrm{T} 1$ presenta un valor de $0.009 \mathrm{mg} / \mathrm{L}$ con una media de $0.016 \mathrm{mg} / \mathrm{L}$ y una desviación estándar de 0.008. En el caso de cianuros la media es de $0.004 \mathrm{mg} / \mathrm{L}$ y para Arsénico la media resulta de $0.002 \mathrm{mg} / \mathrm{L}$ valores muy por debajo del máximo permitido por la NORMA INEN 1180:2014.

Pudiéndose establecer que en el Sector Norte Los Pocitos todas las muestras analizadas cumplen con la NORMA INEN 1108:2014 en lo referente a los Indicadores Químicos arriba mencionados. Sin embargo, resulta particularmente inusual el valor del análisis químico del parámetro Cloro libre residual presentando la muestra T3 el valor más alto con 0.018 ppm y la 
muestra T1 el valor más bajo de 0.10 ppm con una media de $0.14 \mathrm{ppm}$, de modo tal que este valor es menos del $50 \%$ del rango mínimo permitido que es de 0.3 ppm basado en la NORMA INEN 1108:2014. La desviación estándar para nitritos, Cianuro, Arsénico y Cloro Libre Residual se encuentran en el orden de centésimas a milésimas lo que indica que los resultados obtenidos están pocos dispersos

\section{Conclusiones.}

De acuerdo a los resultados obtenidos en los análisis físico químicos del agua potable expendida por Tanqueros en la parroquia El Morro se pudo determinar que todas las muestras arrojaron valores muy por debajo del máximo permitido por la NORMA INEN 1108:2014 para el parámetro Cloro Libre Residual por lo que se considera que el agua distribuida no es apta para el consumo de las personas.

Los resultados microbiológicos de las nueve muestras obtenidas presentan en su totalidad un alto número de Coliformes fecales siendo el sector Norte el más afectado con los valores más elevados; por lo tanto, el agua potable analizada no cumple con el requisito de la NORMA INEN 1108:2014.

La República del Ecuador de conformidad con lo que establece la Constitución como norma jerárquica en su artículo 12, indica el derecho humano al agua que es fundamental e irrenunciable. El agua constituye patrimonio nacional estratégico de uso público, inalienable,imprescriptible, e inembargable y esencial para la vida, por lo tanto ,se entiende que el agua de consumo humano y uso doméstico son aquellos recursos hídricos que requieren de tratamiento convencional para lo cual deberán cumplir con los criterios de calidad establecidos en las normas sobre la base de 
Madeleen P. Cruz-Vargas; Walter E. Mariscal-Santi; Nancy A. Sorroza-Rojas; Nancy E. Villegas-Villao; Frella S. GarcíaLarreta; Raisa S. Mariscal-García

parámetros físicos, químicos y microbiológicos. En consecuencia, el resultado obtenido en la presente investigación no satisface los requerimientos establecidos en la normatividad ecuatoriana

\section{Recomendaciones.}

Realizar una futura investigación sobre la calidad del agua potable en el sector El Morro en la toma de agua donde se abastecen los tanqueros con el fin de determinar la situación del líquido vital en este punto y contrastar con los resultados frutos de esta investigación.

Realizar controles periódicos al sistema de abastecimiento del agua, para garantizar de esta forma que este líquido vital se mantenga inocuo desde la central de distribución hasta los diferentes sitios a los que va destinado, previniendo así alguna posible contaminación a futuro.

Realizar una charla de concientización a la población de la parroquia rural de El Morro sobre el riesgo que corren si consumen el agua que se distribuye en tanqueros, y los problemas que pueden presentarse en su salud si continúan con el consumo de esta agua, así como métodos caseros de desinfección.

Realizar un estudio más profundo del microorganismo Cryptosporidium en agua potable, ya que es un indicador de contaminación poco inusual pero importante.

\section{Bibliografía.}

Aricoché, M. (2012). Sistema de abastecimiento deagua potable para cuatro poblados rurales. Piura: Universidad de Piura.

Arrojo, P. (2006). El reto ético de la nueva cultura del agua. Funciones, valores y derechos en juego. Barcelona: Paidós.

Becerra Ramírez, J., \& Salas Benítez, I. (2016). El derecho humano al acceso al agua potable: aspectos filosóficos y constitucionales de su configuración y garantía en latinoamérica. Revista Prolegómenos - Derechos y Valores, 19(37), 125-146. 
Chiles Arévalo, G. (2015). Evaluación de la calidad y cantidad de agua de las juntas administradoras de agua potable del cantón montúfar para el diseño de un plan de mejoramiento y aprovechamiento adecuado. Ibarra: Universidad Técnica del Norte.

GAD. (2015). PLAN DE DESARROLLO Y ORDENAMIENTO TERRITORIAL El MORRO. Guayaquil.

INEN. (2014). NORMA TÉCNICA ECUATORIANA. Quito.

Ramos Castellanos, P. (2007). Uso eficiente y sostenible de los recursos naturales. Salamanca: Universidad de Salamanca.

Rodriguez, J. (2008). Parámetros fisicoquímicos de dureza total en calcio y magnesio, pH, conductividad y temperatura del agua potable analizados en conjunto con las Asociaciones Administradoras del Acueducto, (ASADAS), de cada distrito de Grecia, cantón de Alajuela. Pensamiento Actual, 9(12). 